\title{
Mixed valence of Sm on metal single-crystal surfaces
}

\author{
J. N. Andersen, I. Chorkendorff, ${ }^{*}$ and J. Onsgaard \\ Fysisk Institut, Odense Universitet, Campusvej 55, DK-5230 Odense M, Denmark \\ J. Ghijsen, ${ }^{\dagger}$ R. L. Johnson, and F. Grey \\ Max Planck Institut für Festkörperforschung, Heisenbergstrasse 1, D-7000 Stuttgart 80, Federal Republic of Germany
}

(Received 30 September 1987)

\begin{abstract}
Results of a photoemission study of $\mathrm{Sm}$ on a $\mathrm{Cu}(100)$ single-crystal surface are reported. From the binding energy of the $\mathrm{Sm} 4 f$ levels it is concluded that the mixed valence of $\mathrm{Sm}$ on $\mathrm{Cu}(100)$ is of a heterogeneous nature. It is suggested that this conclusion also applies to $\mathrm{Al}(111)$ and Al(100).
\end{abstract}

Questions of the surface valency and surface valence transitions of lanthanide metals and intermetallic compounds have been addressed by a number of studies using photoemission. In an early study, ${ }^{1}$ it was concluded that either the bulk or the surface of Sm could very well be in a homogeneous mixed-valence state. Later studies ${ }^{2-6}$ have demonstrated that the mixed valence is of a heterogeneous nature, that is the bulk Sm is trivalent $\left(4 f^{5}\right)$ and the surface divalent $\left(4 f^{6}\right)$ as suggested by Johansson. ${ }^{7}$ Surface valence transitions from a trivalent bulk to a divalent surface have been observed in a number of trivalent lanthanide intermetallic compounds such as $\mathrm{YbAu}_{2}$ (Ref. 8), $\mathrm{SmAl}_{2}$ (Refs. 9 and 10), $\mathrm{YbPd}_{3}$ (Ref. 11), EuPd 3 , and $\operatorname{EuPd}_{5}$ (Ref. 12). It has been demonstrated that the surfaces of several homogeneous mixed divalent-trivalent compounds such as $\mathrm{SmB}_{6}$ (Ref. 4), $\mathrm{YbAl}_{2}$ (Ref. 13), and YbPd (Ref. 11) are divalent. Several studies ${ }^{14-17}$ of thin layers of $\mathrm{Yb}$ evaporated onto single-crystal surfaces of $\mathrm{Al}$ and $\mathrm{Ni}$ showed that the $\mathrm{Yb}$ atoms at the surface were divalent, whereas the $\mathrm{Yb}$ atoms that had diffused into the bulk of the sample became trivalent ${ }^{15-17}$ or had homogeneous mixed valency. ${ }^{14}$ In all of these systems the mixed valence has a heterogeneous nature apart from in the bulk of the homogeneous mixed-valence compounds.

In a recent series of papers by Fäldt and Myers, ${ }^{18-21}$ it has been claimed that thin layers of Sm on single-crystal surfaces of $\mathrm{Cu}$ and $\mathrm{Al}$ exhibit homogeneous mixed valence. In this Rapid Communication we report the results of a photoemission study of the $4 f \mathrm{Sm}$ levels of thin $\mathrm{Sm}$ layers on $\mathrm{Cu}(100)$ single crystals, which prove that the mixed valence of $\mathrm{Sm}$ on $\mathrm{Cu}(100)$ is heterogeneous.

Before describing our experimental results we briefly address the use of photoemission techniques for determining the valence state of $\mathrm{Sm}$ deposited on metal surfaces. Fäldt and Myers ${ }^{18-21}$ made use of the fact that the binding energy of the $\mathrm{Sm} 3 d$ core levels shifts by about $10 \mathrm{eV}$ when going from the divalent to the trivalent state. The ratio between these two peaks was then used for assessing the valence. The claim that the mixed valence of the surface layer was homogeneous and not heterogeneous was based entirely on interpretation of the low-energy electron-diffraction (LEED) patterns that formed and on the assumption that the Sm stayed at the surface and did not alloy. In our opinion, the question whether homogeneous or heterogeneous mixed valence occur cannot be settled by such an analysis of the $3 d \mathrm{Sm}$ levels and the LEED patterns, unless angle-resolved measurements of the $3 d$ emission are performed. ${ }^{2}$ When using the method of Refs. 18-21, the homogeneity of the mixed valence has to be based on geometrical information obtained from other methods, and such unambiguous information is, in general, rather hard to obtain. A more direct way of investigating the possibility of homogeneous mixed valence is to perform a photoemission study of the $4 f$ core levels of the lanthanide. The basic requirement for observing mixed valence is, of course, that emission features from both diand trivalent $\mathrm{Sm}$ are seen. In order for the mixed valence of a layer of $\mathrm{Sm}$ to be homogeneous the energy separation per $\operatorname{Sm}$ atom $\Delta E_{\mathrm{II}, \mathrm{III}}$ between the divalent and the trivalent state of the whole Sm layer has to be close to zero. It has been shown ${ }^{22,23}$ that the energy difference is related to the measured binding energy (referred to the Fermi level) $E_{B}$ of the divalent multiplet term closest to the Fermi level by

$$
\Delta E_{\mathrm{II}, \mathrm{II}}=E_{B}-E_{\mathrm{imp}}^{\text {ip }}
$$

where $E \mathrm{FP}^{\mathrm{p}}$ is the solution energy of a trivalent $\mathrm{Sm}$ atom, that has not geometrically relaxed from its divalent size, in the unperturbed $\mathrm{Sm}$ layer.

Clearly, the impurity term $E \mathrm{Imp}^{\mathrm{p}}$ has to be estimated before using Eq. (1) for evaluating the possibility of homogeneous mixed valence. It has been shown ${ }^{23}$ that in the bulk of a divalent lanthanide metal the impurity term $E$ imp is smaller than $\sim 0.5 \mathrm{eV}$. At the surface this value is expected to be reduced to $\alpha \times 0.5 \mathrm{eV}$ where $\alpha \sim 0.7-0.8$ (Refs. 10 and 23), i.e., to $\sim 0.4 \mathrm{eV}$. In the case of a homogeneous mixed-valence $\mathrm{Sm}$ layer the impurity term will be even smaller; as the valence approaches 3 the impurity term will by definition tend to zero. In the case of lanthanide intermetallic compounds it has further been shown ${ }^{10,23}$ that the equivalent impurity term is very close to zero. In dilute overlayers of Sm on metal single-crystal surfaces the above estimate of $0.4 \mathrm{eV}$ is thus expected to be an overestimate of the impurity term. Stated alternatively, the most relaxed necessary (but certainly not sufficient) condition for homogeneous mixed valence of $\mathrm{Sm}$ on a metal single-crystal surface is that the binding 
energy of the Sm multiplet term closest to the Fermi level is smaller than $0.4 \mathrm{eV}$. If this condition is not satisfied the mixed valence is of heterogeneous type.

The experiments were performed at the Flipper II beamline $^{24}$ at the DESY storage ring DORIS in Hamburg. The $\mathrm{Cu}(100)$ crystal was cleaned by a combination of sputtering and annealing. High-purity $\mathrm{Sm}$ was evaporated onto the $\mathrm{Cu}(100)$ crystal close to room temperature, the amount was determined by using a quartz crystal oscillator. Cleanness of the deposited Sm layers was checked by $x$-ray photoelectron spectroscopy (XPS) and by photoemission with a photon energy of either 141 or 30 eV. The deposited layers were found to be free from contamination; special attention was paid to oxygen which, if present, should have shown up clearly in the $30 \mathrm{eV}$ photoemission spectra. The emitted photoelectrons were collected and energy analyzed by a double-pass cylindrical mirror analyzer (CMA) operated in the constant pass en-

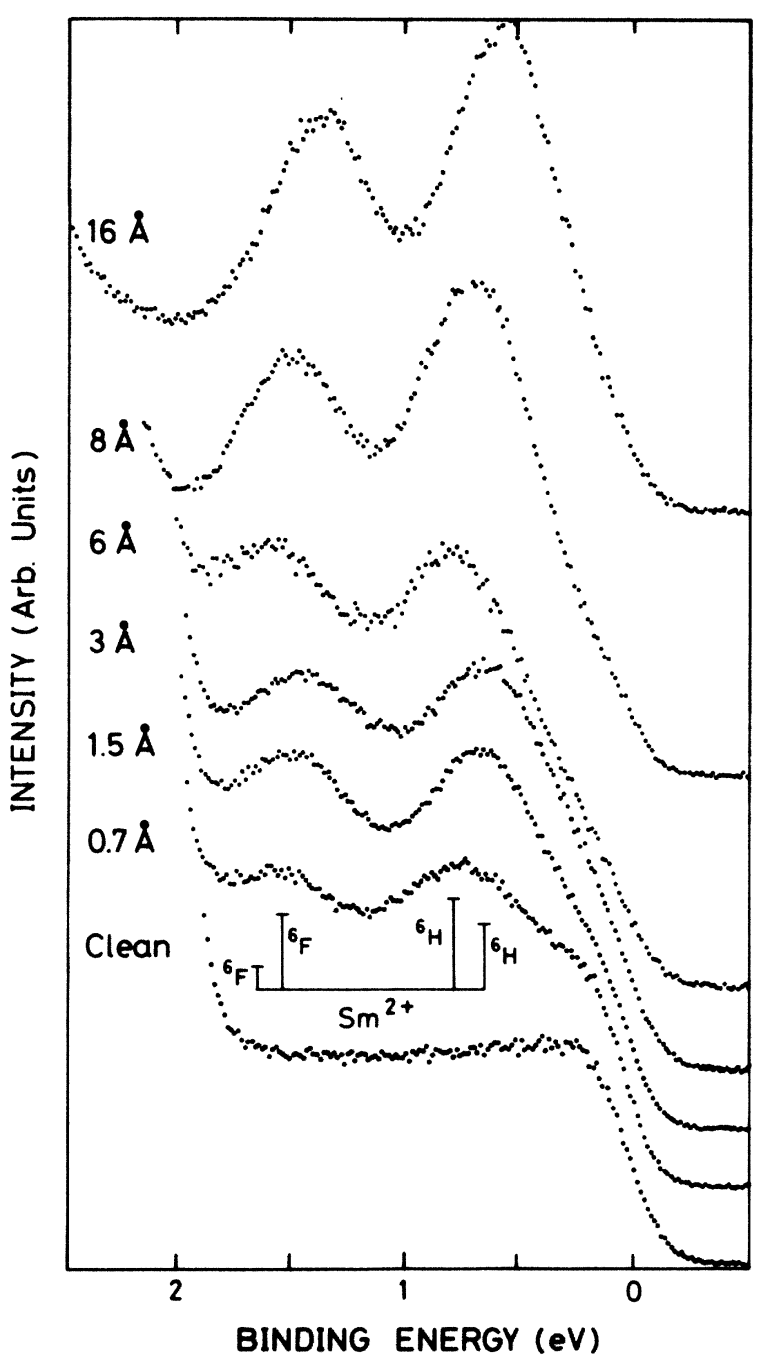

FIG. 1. Photoemission spectra of the region close to the Fermi level for clean and $\mathrm{Sm}$-covered $\mathrm{Cu}(100)$ surfaces. $h v=60$ eV. The extra features at low binding energy are due to emission from divalent $\mathrm{Sm}$. The divalent Sm multiplet pattern (Ref. 28 ) is shown at an energy corresponding to the $0.7 \AA$ coverage. ergy mode. The angle between the surface normal and the CMA axis was $10 \mathrm{deg}$. Photoemission spectra of the Sm overlayers were measured at two photon energies, 60 and $141 \mathrm{eV}$. At $141 \mathrm{eV}$ both the di- and the trivalent Sm shown resonantly enhanced photoemission ${ }^{4}$ leading to an increased sensitivity for $\mathrm{Sm}$ at this photon energy. At 141 $\mathrm{eV}$ a somewhat higher bulk sensitivity is obtained than in the $60-\mathrm{eV}$ photon energy measurements. The $60-\mathrm{eV}$ photon energy was chosen as a compromise between resolution and cross section of the Sm $4 f$ levels.

Figures 1 through 3 show representative photoemission spectra of the clean and of Sm-covered $\mathrm{Cu}(100)$ surfaces. On the high binding-energy side of the $\mathrm{Cu} 3 d$ band the final-state multiplet pattern of trivalent $\mathrm{Sm}$ is seen in Figs. 2 and 3. In the region between the $\mathrm{Cu} 3 d$ band and the Fermi level the emission features of the divalent Sm appear. A weak divalent Sm feature at about $4 \mathrm{eV}$ binding energy is totally obscured by the strong $\mathrm{Cu} 3 d$ emission.

It can be seen from Fig. 1 that the peak originating from the divalent multiplet terms closest to the Fermi level has a binding energy of about $0.55 \mathrm{eV}$ for all coverages shown. Towards low coverage this binding energy is seen

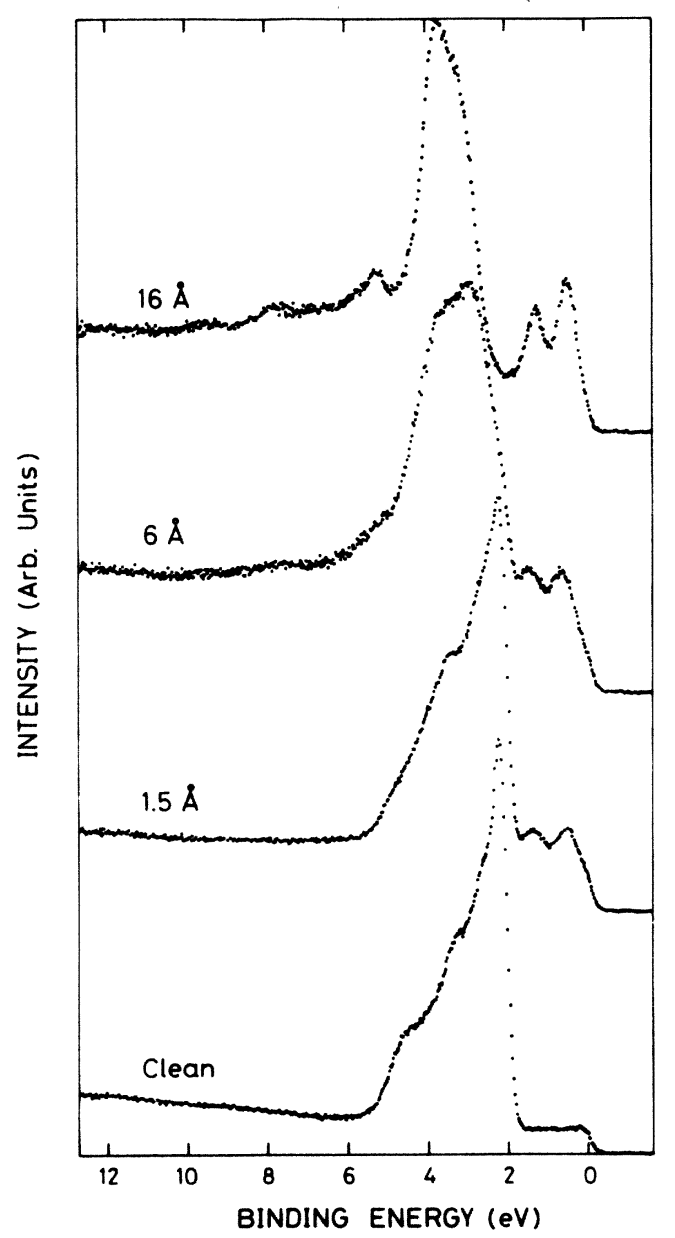

FIG. 2. Photoemission spectra of the valence region for clean and $\mathrm{Sm}$-covered $\mathrm{Cu}(100)$ surfaces. $h v=60 \mathrm{eV}$. The extra features at lower binding energy than the $\mathrm{Cu} 3 d$ are due to divalent Sm, those at higher binding energy to trivalent Sm. 


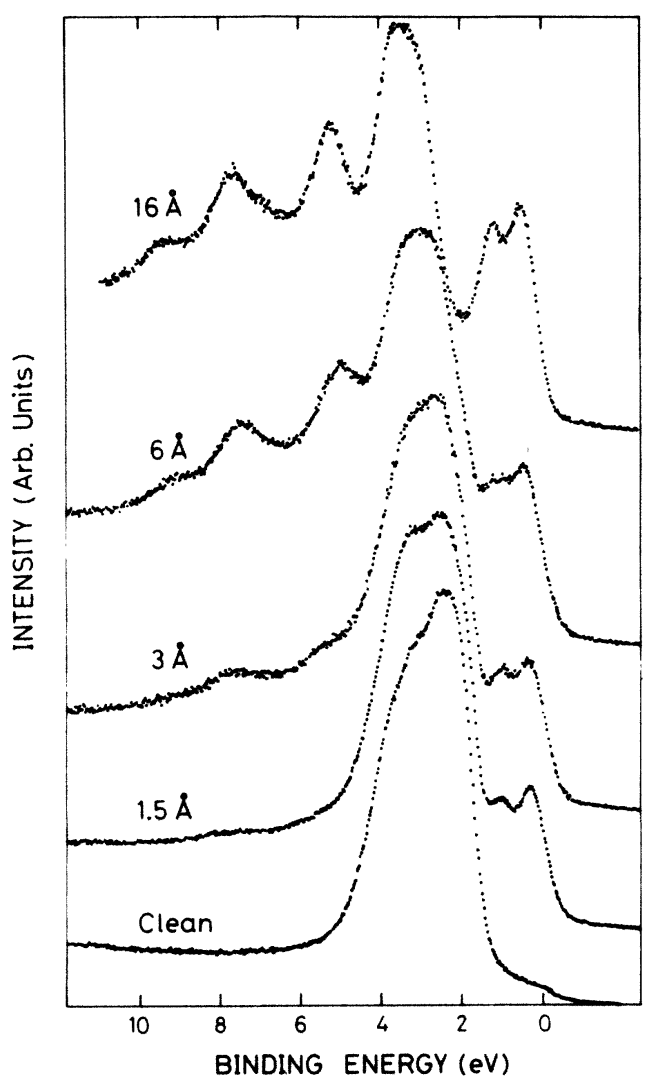

FIG. 3. As Fig. 2 but $h v=141 \mathrm{eV}$.

to increase slightly as is to be expected for a layer of isolated $\mathrm{Sm}$ atoms. The increase in $\mathrm{Sm} 4 f$ binding energy between 3 and $6 \AA$ coverage indicates the formation of either a $\mathrm{Sm}-\mathrm{Cu}$ alloy or a pure $\mathrm{Sm}$ layer underneath the divalent surface Sm. Similar behavior has been observed for the $\mathrm{Yb} / \mathrm{Ni}(100)$ system where a compound layer develops. ${ }^{16,17,25}$

The measured binding energy of the highest-lying multiplet term does not meet even the very relaxed requirement for homogeneous mixed valence of being smaller than $0.4 \mathrm{eV}$. It should further be noticed that the $60-\mathrm{eV}$ photon energy spectra of Fig. 2 show only small amounts of trivalent Sm. If the surface layer of Sm was in a homogeneous mixed-valence state with a valence of larger than say 2.5 , as is claimed for large coverages in Ref. 19, a much larger trivalent signal should result. We must conclude, therefore, that the mixed valence is heterogeneous and not homogeneous in contrast with the claim of Ref. 19.

The heterogeneity of the mixed valence is most likely caused by an intermixing between the $\mathrm{Sm}$ and the $\mathrm{Cu}$ substrate leading to a divalent surface layer and a trivalent bulk layer of $\mathrm{Sm}$ that has reacted with $\mathrm{Cu}{ }^{26}$ At large coverages also the growth of a clean $\mathrm{Sm}$ overlayer with a trivalent bulk and a divalent surface on top of the interface could very well occur. Such behavior has been observed in similar systems ${ }^{14-17}$ and is also expected from theoretical considerations. ${ }^{10,22,23}$ The large increase in the ratio between the divalent and the trivalent signals when changing the photon energy from $141 \mathrm{eV}$ to the more surface-sensitive $60 \mathrm{eV}$, Figs. 2 and 3, seems to support this interpretation. However, it should be kept in mind that the resonance of the $4 f$ emission at $141 \mathrm{eV}$ changes the ratio between the di- and trivalent cross sections which to some extent will interfere with the mean-free-path considerations. Further, one of the LEED patterns observed suggests that a reaction occurs. At $6 \AA$ coverage the LEED pattern is that of a slightly distorted hexagonal structure with two orthogonal domains (we believe this to be the hexagonal structure reported in Ref. 19) a structure which is also seen on $\mathrm{Yb} / \mathrm{Ni}(100)$ (Refs. 16 and 17) under conditions where reaction has occurred. Also, the narrowing and shift to higher binding energy of the $\mathrm{Cu} 3 d$ band suggest that a reaction has occurred between the $\mathrm{Sm}$ and the $\mathrm{Cu}$. Finally we note that little trivalent $\mathrm{Sm}$ is seen in the spectra of Fig. 3 for coverages below $3 \AA$ indicating that a certain threshold coverage has to be reached before reaction starts to occur, as in the $\mathrm{Yb} / \mathrm{Ni}(100)$ system. The existence of such a threshold coverage has also been reported for Sm on semiconductor surfaces. 27

We believe that the same conclusion concerning the heterogeneity of the mixed valence of the $\mathrm{Sm}$ layers also applies in the case of Sm on Al(111). An ultraviolet photoemission spectroscopy (UPS) spectrum of $\mathrm{Sm}$ on Al(111) showing the region close to the Fermi level [Fig. 6(c) in Ref. 21] reveals that the binding energy of the Sm multiplet term closest to the Fermi level is larger than $\mathbf{0 . 7}$ eV. Hence, the requirement for homogeneous mixed valence is not satisfied in this case either.

Assuming that intermixing between $\mathrm{Sm}$ and the substrate is the cause of the heterogeneous mixed valence also in the case of $\mathrm{Sm}$ on $\mathrm{Al}$ substrates would explain some of the findings of Refs. 18, 20, and 21. In the case of $\mathrm{Al}(111)$ it is reported ${ }^{21}$ that thick (3-10 monolayers) annealed films of $\mathrm{Sm}$ always exhibit the valence 3 instead of about 2.6 as would be expected from a thin $\mathrm{Sm}$ film. ${ }^{2}$ For submonolayer annealed films it was found that $\mathrm{Sm}$ is trivalent. ${ }^{21}$ The authors state that they are unable to explain this result, but the problem is due to their assumption of no intermixing. Clearly, these results could be explained by. a reaction between the $\mathrm{Sm}$ and the $\mathrm{Al}(111)$ substrate, the annealing being necessary in order to provide sufficient mobility. In the case of $\mathrm{Sm}$ on $\mathrm{Al}(100)$ (Refs. 18 and 20) mainly trivalent $\mathrm{Sm}$ is observed after annealing. Furthermore, it is reported that the annealing of thick Sm films leads to a reappearance of the $\mathrm{Al} 2 p$ XPS peaks and to a reduction of the $\mathrm{Sm} 3 d$ intensity which is explained as being due to an agglomeration of the Sm film. However, exactly the same behavior would result if intermixing took place. Such interdiffusion would explain the increased excitation of bulk plasmons by the emitted Sm $3 d$ photoelectrons in annealed films compared to unannealed ones. ${ }^{20}$

In summary, we have demonstrated that the mixed valence of $\mathrm{Sm}$ on $\mathrm{Cu}(100)$ is of heterogeneous type. Based on a published ${ }^{21}$ UPS spectrum the same conclusion was reached for the mixed valence of $\mathrm{Sm}$ on $\mathrm{Al}(111)$ and we suggest that the same also applies for Sm on $\mathrm{Al}(100)$. The heterogeneity results from a divalent surface layer and a trivalent intermetallic compound. 
Many stimulating discussions with N. Mårtensson and A. Nilsson are gratefully acknowledged, as is financial support of this work by the Danish Natural Science Research Council and the German Federal Minister for Research and Technology (BFMT) under Project No. 05390 CAB.

*Permanent address: Laboratory of Applied Physics II, Technical University of Denmark, DK-2800 Lyngby, Denmark.

†Present address: Technical Physics Laboratory, University of Groningen, Nijenborgh 18, NL-9747 AG Groningen, The Netherlands.

${ }^{1}$ G. K. Wertheim and M. Campagna, Chem. Phys. Lett. 47, 182 (1977).

${ }^{2}$ G. K. Wertheim and G. Crecelius, Phys. Rev. Lett. 40, 813 (1978).

3J. K. Lang and Y. Baer, Solid State Commun. 31, 945 (1979).

${ }^{4}$ J. W. Allen, L. I. Johansson, I. Lindau, and S. B. M. Hagström, Phys. Rev. B 21, 1335 (1980).

5 J. K. Lang, Y. Baer, and P. A. Cox, J. Phys. F 11, 121 (1981).

${ }^{6}$ F. Gerken, A. S. Flodström, J. Barth, L. I. Johansson, and C. Kunz, Phys. Scr. 32, 43 (1985).

${ }^{7}$ B. Johansson, in Rare Earths and Actinides 1977, edited by W. D. Corner and B. K. Tanner, Institute of Physics Conference Series, Vol. 37 (IOP, Bristol, 1978), p. 39.

${ }^{8}$ G. K. Werteim, J. H. Wernick, and G. Crecelius, Phys. Rev. B 18, 875 (1978).

${ }^{9}$ G. Kaindl, W. D. Schneider, C. Laubschat, B. Reihl, and N. Mårtensson, Surf. Sci. 126, 105 (1983).

${ }^{10}$ C. Laubschat, G. Kaindl, W. D. Schneider, B. Reihl, and N. Mårtensson, Phys. Rev. B 33, 6675 (1986).

${ }^{11}$ M. Domke, C. Laubschat, E. V. Sampathkuraman, M. Prietch, T. Mandel, and G. Kaindl, Phys. Rev. B 32, 8002 (1985).

${ }^{12}$ V. Murgai, L. C. Gupta, R. D. Parks, N. Mårtensson, and B. Reihl, in Valence Instabilities, edited by P. Wachter and H. Boppart (North-Holland, Amsterdam, 1982), p. 299.

${ }^{13}$ G. Kaindl, B. Reihl, D. E. Eastman, R. A. Pollak, N. Mårtensson, B. Barbara, T. Penney, and T. S. Plaskett, Solid State Commun. 41, 157 (1982).

${ }^{14}$ R. Nyholm, I. Chorkendorff, and J. Schmidt-May, Surf. Sci. 143, 177 (1984).
${ }^{15}$ I. Chorkendorff, J. Onsgaard, J. Schmidt-May, and R. Nyholm, Surf. Sci. 160, 587 (1985).

${ }^{16} \mathrm{~J}$. N. Andersen, J. Onsgaard, A. Nilsson, B. Eriksson, E. Zdansky, and N. Mårtensson, Surf. Sci. 189/190, 399 (1987).

${ }^{17}$ A. Nilsson, B. Eriksson, N. Mårtensson, J. N. Andersen, and J. Onsgaard, Phys. Rev. B 36, 9308 (1987).

${ }^{18} \AA$. Fäldt and H. P. Myers, Solid State Commun. 48, 253 (1983).

${ }^{19} \AA$. Fäldt and H. P. Myers, Phys. Rev. Lett. 52, 1315 (1984).

${ }^{20} \AA$. Fäldt and H. P. Meyers, Phys. Rev. B 30, 5481 (1984).

${ }^{21} \AA$. Fäldt and H. P. Myers, Phys. Rev. B 34, 6675 (1986).

22B. Johansson, Phys. Rev. B 20, 1315 (1979).

${ }^{23}$ B. Johansson and N. Mårtensson, in Handbook on the Physics and Chemistry of Rare Earths, edited by K. A. Gschneidner, Jr., L. Eyring, and S. Hüfner (North-Holland, Amsterdam, 1987), Vol. 10.

${ }^{24}$ R. L. Johnson and J. Reichardt, Nucl. Instrum. Methods 208, 791 (1983).

${ }^{25}$ A. Nilsson, B. Eriksson, N. Mårtensson, J. N. Andersen, and J. Onsgaard (unpublished).

${ }^{26} \mathrm{Sm}-\mathrm{Cu}$ alloys are expected to be trivalent in the bulk. This follows from the fact that the unit-cell volume of the alloys shows no deviation from the trend of other trivalent lanthanide-Cu alloys. (See Ref. 8 for $\mathrm{SmCu}_{2}$.) Furthermore, the trivalency of the $\mathrm{Sm}$ is expected from the valence stability considerations of Ref. 23 and the knowledge that both di- and trivalent $\mathrm{Yb}-\mathrm{Cu}$ alloys exist [A. Iandelli and $\mathrm{A}$. Palenzona, in Handbook on the Physics and Chemistry of Rare Earths, edited by K. A. Gschneidner, Jr. and L. Eyring (NorthHolland, Amsterdam, 1979), Vol. 2].

${ }^{27}$ M. Grioni, J. J. Joyce, and J. H. Weaver, Phys. Rev. B 32, 962 (1985).

${ }^{28}$ F. Gerken, J. Phys. F 13, 703 (1983). 